Article

\title{
Near- and Far-Field Excitation of Topological Plasmonic Metasurfaces
}

\author{
Matthew Proctor ${ }^{1, *(D)}$, Xiaofei Xiao ${ }^{2}\left(\mathbb{D}\right.$, Richard V. Craster ${ }^{1} \mathbb{D}$, Stefan A. Maier ${ }^{2,3} \mathbb{I D}^{\text {, }}$ \\ Vincenzo Giannini 4 (D) and Paloma Arroyo Huidobro ${ }^{5}$ (D) \\ 1 Department of Mathematics, Imperial College London, London SW7 2AZ, UK; r.craster@imperial.ac.uk \\ 2 Blackett Laboratory, Department of Physics, Imperial College London, London SW7 2AZ, UK; \\ xiaofei.xiao15@imperial.ac.uk (X.X.); s.maier@imperial.ac.uk (S.A.M.) \\ 3 Chair in Hybrid Nanosystems, Nano-Institute Munich, Faculty of Physics, \\ Ludwig-Maximilians-Universität München, 80539 München, Germany \\ 4 Instituto de Estructura de la Materia (IEM), Consejo Superior de Investigaciones Científicas (CSIC), \\ Serrano 121, 28006 Madrid, Spain; v.giannini@csic.es \\ 5 Instituto de Telecomunicações, Insituto Superior Tecnico-University of Lisbon, Avenida Rovisco Pais 1, \\ 1049-001 Lisboa, Portugal; p.arroyo-huidobro@lx.it.pt \\ * Correspondence: matthew.proctor12@imperial.ac.uk
}

Received: 21 August 2020; Accepted: 21 September 2020; Published: 24 September 2020

\begin{abstract}
The breathing honeycomb lattice hosts a topologically non-trivial bulk phase due to the crystalline-symmetry of the system. Pseudospin-dependent edge states, which emerge at the interface between trivial and non-trivial regions, can be used for the directional propagation of energy. Using the plasmonic metasurface as an example system, we probe these states in the nearand far-field using a semi-analytical model. We provide the conditions under which directionality was observed and show that it is source position dependent. By probing with circularly-polarised magnetic dipoles out of the plane, we first characterise modes along the interface in terms of the enhancement of source emissions due to the metasurface. We then excite from the far-field with non-zero orbital angular momentum beams. The position-dependent directionality holds true for all classical wave systems with a breathing honeycomb lattice. Our results show that a metasurface in combination with a chiral two-dimensional material, could be used to guide light effectively on the nanoscale.
\end{abstract}

Keywords: topological nanophotonics; plasmonic metasurface; edge states; pseudospin; chiral

\section{Introduction}

Topological nanophotonics offers a path towards efficient and robust control over light on the nanoscale [1]. Concepts borrowed from topological insulators, materials which host protected surface states for electrons, can also be applied to photonic systems. Following theoretical proposals [2,3], protected photonic modes, reliant on a explicit time-reversal symmetry breaking component, have been demonstrated experimentally [4]. However, these require strong magnetic fields or complex materials with a large magneto-optical response, which makes such systems difficult to miniaturise. More recently, these effects have been proposed using graphene $[5,6]$, which naturally has a large magneto-optical response. This is limited to the infrared regime, however, which restricts potential applications. Methods of achieving topological protection through the crystalline symmetry of a system whilst preserving time-reversal symmetry are therefore appealing, since they do not require complex setups and are not restricted to a specific frequency regime. These effects fall into two main categories: valley effects, which rely on extrema in the band structures of materials [7-9], 
and pseudospin-dependent effects, which rely on the spin angular momentum texture of the electromagnetic fields [10,11].

First proposed in [12], the pseudospin-dependent effect relies on a triangular lattice of hexamers to produce states reminiscent of the quantum spin Hall effect (QSHE) in topological insulators. This breathing honeycomb lattice has two gapped phases: the shrunken phase, where the hexamers are perturbed inwards, and the expanded phase, where they are perturbed outwards. Despite both having a trivial $\mathbb{Z}_{2}$ index, the phases of the breathing honeycomb are topologically distinct-while the shrunken phase is a trivial insulator, the expanded one is an instance of an "obstructed atomic limit" phase $[13,14]$, and edge states will appear between regions in either phase. A direct analogy of the QSHE would produce purely unidirectional edge states, in the absence of spin-mixing impurities. However, as we showed in a previous work [15], the edge mode directionality for near-field sources is source-position-dependent and is determined by the spin angular momentum of the modes: it is the local handedness of the elliptical field polarisation which determines the propagation direction of the edge modes, rather than an absolute pseudospin quantity as in the QSHE [15]. This result is true for any bosonic breathing honeycomb lattice [16], since it is rooted in time-reversal symmetry and the absence of Kramer's degeneracy for bosons as opposed to fermions. There have been a range of experimental investigations on the breathing honeycomb photonic crystal [17-20], including the observation of edge modes in the visible regime [21,22]. Despite this, comprehensive theoretical studies of the directional excitation of pseudospin edge modes in the breathing honeycomb lattice based on an analysis of their inhomogeneous electromagnetic angular momentum are scarce; in particular, modelling with a circularly polarised incident beam, as used in recent experiments $[17,21,22]$ are needed.

In this article, we consider a plasmonic metasurface consisting of a two-dimensional array of metallic nanoparticles with a breathing honeycomb lattice. We first characterise the optical response of the bulk modes and then investigate the propagation properties of edge states which emerge at the interface between trivial and and non-trivial regions. We go beyond our previous work and extend the understanding of excitation by near-field sources by probing edge states with sources out of the plane. Furthermore, for the first time we theoretically characterise the directionality behaviour with far-field beams. We show that, for this regime, the propagation direction of the edge mode is determined by the interplay between beam polarisation and the electric field phase of the edge eigenmodes. With this, we unambiguously show that the position of the source or beam is critical in exciting a unidirectional mode with the breathing honeycomb lattice.

Whilst the results we present are in the plasmonic metasurface, we emphasise that the properties and behaviour of the edge states are valid for any classical wave system.

\section{Methods}

We model the system of subwavelength metallic nanoparticles (NPs) using the coupled dipole method. When the nearest neighbour spacing $R$ and NP radius $r$ satisfied $R>3 r$, each NP can be treated as a point dipole $[23,24]$. To model nanorods, we use spheroidal NPs with a radius $r=10 \mathrm{~nm}$ and height $h=40 \mathrm{~nm}$. For NPs of this size and shape, it is necessary to include depolarization and radiative effects, so we use the Meier-Wokaun long wavelength approximation (MWLWA) to describe the the dipolar optical response of an individual NP $[25,26]$. The MWLWA polarisability $\alpha(\omega)$ for spheroids is,

$$
\alpha(\omega)=\frac{\alpha_{S}(\omega)}{1-\frac{k^{2}}{l_{E}} D \alpha_{S}(\omega)-i \frac{2 k^{3}}{3} \alpha_{S}(\omega)},
$$

with the static polarisability $\alpha_{s}(\omega)$,

$$
\alpha_{s}(\omega)=\frac{V}{4 \pi} \frac{\epsilon(\omega)-1}{1+L(\epsilon(\omega)-1)} .
$$


$k=\epsilon_{m} \omega / c$ is the wave number and the environment is a homogeneous vacuum with $\epsilon_{m}=1 . V$ is the $\mathrm{NP}$ volume and $D$ and $L$ are dynamic and static geometrical factors, and $l_{E}$ is the spheroid half-axis; $D=1, L=1 / 3$ and $l_{E}=r$ for a sphere. The dielectric function $\epsilon(\omega)$ is given by the Drude model,

$$
\epsilon(\omega)=\epsilon_{\infty}-\frac{\omega_{p}^{2}}{\omega^{2}+i \omega \gamma}
$$

We use silver NPs, with $\epsilon_{\infty}=5, \omega_{p}=8.9 \mathrm{eV}$ and $\gamma=1 / 17 \mathrm{fs} \approx 38 \mathrm{meV}$ [27]. For a system of multiple NPs, we write a self-consistent coupled dipole equation, which describes the dipole moment of a NP due to neighbouring NPs as well as an incident electric field $\mathbf{E}_{\text {inc, }}$

$$
\frac{1}{\alpha(\omega)} \mathbf{p}_{i}=\mathbf{E}_{\mathrm{inc}}+\hat{\mathbf{G}}\left(\mathbf{d}_{i j}, \omega\right) \cdot \mathbf{p}_{j}
$$

The interactions between dipoles are characterised by the dyadic Green's function [28],

$$
\hat{\mathbf{G}}\left(\mathbf{d}_{i j}, \omega\right)=k^{2} \frac{e^{i k d}}{d}\left[\left(1+\frac{i}{k d}-\frac{1}{k^{2} d^{2}}\right) \hat{\mathbf{I}}-\left(1+\frac{3 i}{k d}-\frac{3}{k^{2} d^{2}}\right) \mathbf{n} \otimes \mathbf{n}\right],
$$

where $\mathbf{d}_{i j}$ is the separation between dipole $i$ and $j, d=\left|\mathbf{d}_{i j}\right|$ and $\mathbf{n}=\mathbf{d}_{i j} / d$. (The $\hat{\cdot}$ represents a dyadic operator.) We only take the zz-component of the Green's function, which corresponds to interactions between dipole moments perpendicular to the separation between the NPs (i.e., out-of-plane). This is a valid approximation since we use spheroidal NPs, causing the in-plane modes to become well separated in frequency from the out-of-plane modes [29]. For a periodic array of NPs in a plasmonic metasurface, we can apply Bloch's theorem and write the following system of equations,

$$
\left(\hat{\mathbf{I}} \frac{1}{\alpha(\omega)}-\hat{\mathbf{H}}\left(\mathbf{k}_{\|}, \omega\right)\right) \cdot \mathbf{p}=\mathbf{E}_{\text {inc }} .
$$

The vector $\mathbf{p}$ contains the out-of-plane dipole moments $p_{z}$ of all NPs in the unit cell. The interaction matrix $\hat{\mathbf{H}}\left(\mathbf{k}_{\|}, \omega\right)$ has elements,

$$
H_{p q}=\left\{\begin{array}{ll}
\sum_{\mathbf{R}} \hat{\mathbf{G}}\left(\mathbf{d}_{p q}+\mathbf{R}, \omega\right) e^{i \mathbf{k}_{\|} \cdot \mathbf{R}} & p \neq q \\
\sum_{|\mathbf{R}| \neq 0} \hat{\mathbf{G}}(\mathbf{R}, \omega) e^{i \mathbf{k}_{\|} \cdot \mathbf{R}} & p=q
\end{array},\right.
$$

where $p, q$ are unit cell indices. The summations run over the lattice sites $\mathbf{R}=n \mathbf{a}_{1}+m \mathbf{a}_{2}$, with lattice vectors $\mathbf{a}_{1}$ and $\mathbf{a}_{2}$. The interaction matrix has dimension $N \times N$ where $N$ is the number of NPs in the unit cell. Since the lattice sums are slowly converging, we use Ewald's method to calculate them $[30,31]$. When modelling plasmonic metasurfaces, it is necessary to include the long range, retarded interactions in the Green's function in Equation (5). Whilst the quasistatic approximation can be used to model very subwavelength plasmonic chains and arrays [32,33], it fails to accurately capture the behaviour of modes at the light line and the radiative broadening and redshifting of modes, which becomes apparent in NPs with $r>10 \mathrm{~nm}$. Additionally, retarded interactions can affect the topological properties of some plasmonic systems [34,35].

We calculate dipole moments by varying $\omega$ and $\mathbf{k}_{\|}$for an incident field $\mathbf{E}_{\text {inc }}$ using Equation (6). We then calculate the extinction cross section $\sigma_{\text {ext }}$ using the optical theorem,

$$
\sigma_{\text {ext }}=\frac{k \operatorname{Im}\left(\mathbf{p} \cdot \mathbf{E}_{\text {inc }}^{*}\right)}{\left|\mathbf{E}^{2}\right|} \text {. }
$$

The incident field satisfies Maxwell's equations, $\left|\mathbf{k}_{\|}\right| E_{\|}+k_{z} E_{z}=0$ (we assume $E_{\|}=1$ and harmonic time dependence $\left.e^{-i \omega t}\right)$. When the field is propagating, above the light line, $k_{z}=\sqrt{k^{2}-\left|\mathbf{k}_{\|}\right|^{2}}$ 
and when it is evanescent, below the light line, $k_{z}=i \sqrt{\left|\mathbf{k}_{\|}\right|^{2}-k^{2}}$. We can also solve Equation (6) as an eigenvalue problem and calculate the spectral function, by letting $\mathbf{E}_{\mathrm{inc}}=0$ and again varying $\omega$ and $\mathbf{k}_{\|}$, which allows us to probe modes whether they are bright or dark. The spectral function $\sigma_{\mathrm{sf}}$ is,

$$
\sigma_{\mathrm{sf}}=k \operatorname{Im}\left(\alpha_{\mathrm{eff}}\right),
$$

with the effective polarisability $\alpha_{\text {eff }}=\sum_{i} 1 / \lambda^{(i)}$ for eigenvalues $\lambda^{(i)}$ [33]. Finally, the coupled dipole method can also be applied to finite systems to model electromagnetic scattering [36].

\section{Optical Response of Bulk and Edge States}

We begin by characterising the optical response of the periodic system, in order to later excite the finite system at the correct frequencies. The breathing honeycomb lattice setup is shown in Figure 1a, with a unit cell with six NPs [12]. In a honeycomb lattice, $R=R_{0}=a_{0} / 3$, where $R$ is the nearest neighbour spacing, and $a_{0}$ is the lattice constant. We let $R_{0}=40 \mathrm{~nm}$, so the lattice constant $a_{0}=120 \mathrm{~nm}$. The larger unit cell (compared to the rhombic Wigner-Seitz unit cell) folds the Brillouin zone and results in a double Dirac cone at $\Gamma$ (as shown in Appendix A, Figure A1). We note that the Dirac cone lies below the localised surface plasmon frequency $\omega_{\text {lsp }}$ due to chiral symmetry breaking and long-range interactions [15]. By perturbing the nearest neighbour separation with a scale factor $s$, such that $R=s R_{0}$, a band gap is opened at $\Gamma$. In Figure $1 \mathrm{c}$, d we plot the extinction cross section $\sigma_{\text {ext }}$ across the Brillouin zone for the shrunken $\left(R=0.9 R_{0}\right)$ and expanded $\left(R=1.065 R_{0}\right)$ lattices. The scale factors are chosen to ensure that the size of the band gaps for the two lattices are approximately equal. We plot the bands from the spectral function as blue dots on top of the extinction cross section as a guide. Below the light line (white dotted line), where modes are confined, the modes have high quality factors, whereas above the light line the modes are very broad, corresponding to larger radiative losses.

In the shrunken lattice, for $\mathbf{k}_{\|}$close to $\Gamma$, the NPs in the unit cell hybridise to form hexapoles, quadrupoles, dipoles and monopoles (from lowest energy to highest energy). The monopolar mode has dipole moments in phase, forming a bonding mode in the out-of-plane dipole moments across the whole lattice. As a result, it couples very strongly with the light line and exhibits a polariton-like splitting. We note that the ordering in this plasmonic metasurface is opposite to the photonic crystal due to the metallic nature of the NPs [15]. A band inversion occurs around $\Gamma$ between the shrunken and expanded phases, causing the dipolar and quadrupolar bands to flip. This is similar to the band inversion process in the QSHE. Although the bands become much broader above the light line, there is still a clear signature between shrunken and expanded phases, which is observable in the far-field, as has been shown previously $[15,37]$. We plot $\sigma_{\text {ext }}$ for a fixed wavevector $\mathbf{k}_{\|}$in Figure 1e, showing how the dipolar peak shifts between the two phases. The broad peak at the top corresponding to the monopolar mode does not shift. Unlike the QSHE, neither the shrunken or expanded lattices have a non-trivial $\mathbb{Z}_{2}$-invariant. Whilst it is possible to define an invariant by taking applying a perturbation theory about $\Gamma$ [12], when characterising the topological properties of the system, it is important to analyse all connected bands in a system. One way of doing this is with the application of topological quantum chemistry. The two phases of the system are still topologically distinct and are distinguishable by their Wilson loops [38]. The shrunken lattice is topologically trivial, whilst the expanded lattice is in an photonic "obstructed atomic limit" phase [13]; it is the $C_{6}$ symmetry which provides the topological character. 
(a)

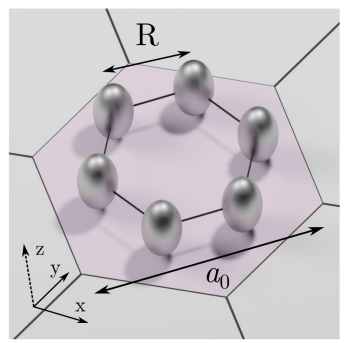

(b)

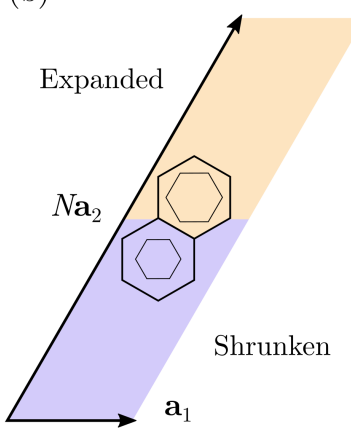

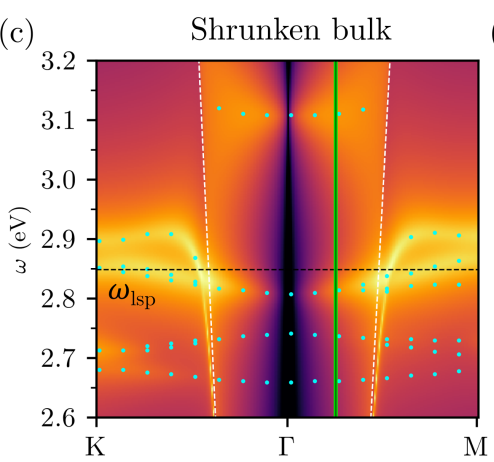

(d)

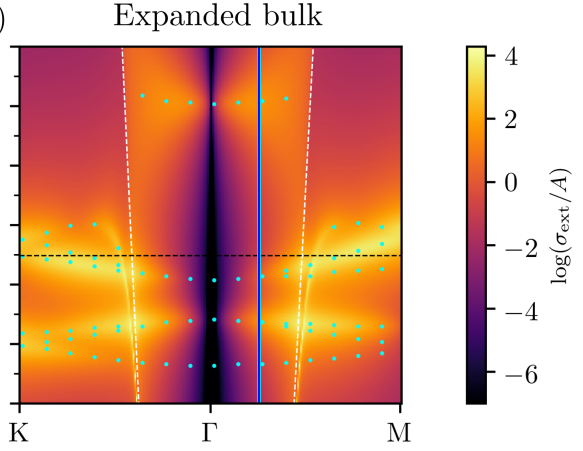

(e)

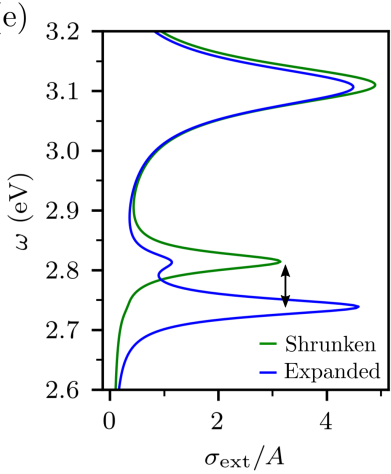

(f)

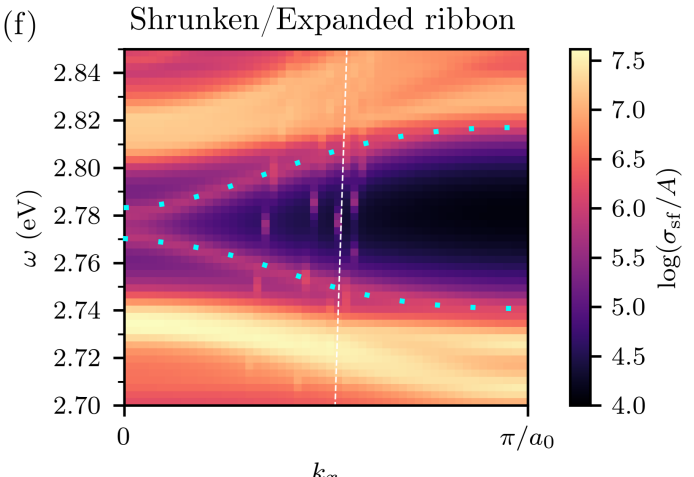

$k_{x}$

Figure 1. Lattice set up and optical response: (a) Unit cell with lattice vectors $\mathbf{a}_{1}=\left(a_{0}, 0\right)$ and $\mathbf{a}_{2}=\left(a_{0} / 2, \sqrt{3} / 2 a_{0}\right)$, the lattice constant $a_{0}=120 \mathrm{~nm}$. The honeycomb lattice has nearest neighbour spacing $R=R_{0}=a_{0} / 3$. The expanded lattice has $R=1.065 R_{0}$ and the shrunken lattice $R=0.9 R_{0}$. (b) Ribbon interface between expanded (orange) and shrunken (blue) regions. The ribbon is doubly periodic, with lattice vectors $\mathbf{a}_{1}$ and $N \mathbf{a}_{2}$. The total ribbon length is $N=20$, with 10 expanded and 10 contracted unit cells. Extinction cross sections $\sigma_{\text {ext }}$ for (c) shrunken and (d) expanded lattices. The band structures, found from the spectral function, are highlighted in blue. (e) $\sigma_{\text {ext }}$ for the shrunken and expanded lattices at $\mathbf{k}_{\|}$, shown in (c,d) (Green and blue vertical lines). A band inversion occurs near $\Gamma$, causing the dipolar band to switch with the quadrupolar band (arrow). (f) Spectral function $\sigma_{\text {sf }}$ for the ribbon interface in (e). Drude losses are $\gamma=10 \mathrm{meV}$ to increase the visibility of the edge states and they are highlighted (blue dots).

Despite having trivial $\mathbb{Z}_{2}$-invariants, when regions in the shrunken phase are placed next to regions in the expanded phase, edge states will appear in the band gap due to the topological origin of the band inversion [15]. These edge states are not topologically protected but do have a pseudospin character, which allows directional modes to be excited through a similar mechanism to the chiral light matter interactions in photonic crystals [39]. We model the edge states of the system by setting up a ribbon with an interface between the two phases, as shown in Figure $1 \mathrm{~b}$. The ribbon supercell is $N=20$ unit cells along the $\mathbf{a}_{2}$ direction, with 10 unit cells in the expanded and shrunken phases, respectively. In Figure 1f, we plot the spectral function $\sigma_{\text {sf }}$ for the ribbon for $\mathbf{k}_{\|}=0$ to $\pi / a_{0}$, with $\gamma=10 \mathrm{meV}$, and we include plots for $\gamma=38 \mathrm{meV}$ in Appendix B, Figure A2. (The spectral function from $\mathbf{k}_{\|}=0$ to $-\pi / a_{0}$ is identical.) Edge states appear in the band gap from $\omega \approx 2.74 \mathrm{eV}$ to $2.81 \mathrm{eV}$ and a small minigap appears between $\omega \approx 2.77 \mathrm{eV}$ to $2.78 \mathrm{eV}$. The mini-gap appears due to the interface breaking $C_{6}$ symmetry, which is the symmetry that protects the topological phase. We note that we use an "armchair" interface here but it is also possible to define other terminations for this lattice [40], including the "zig-zag" interface [11]. In the latter case, the $C_{6}$ symmetry breaking across the interface is slightly smaller and results in a smaller mini-gap, but otherwise the behaviour of the edge states is qualitatively similar. This is evident in the hybridisation of NPs close to the interface: the lower band has an anti-bonding character whilst the upper band has a bonding character (we show these in Appendix C, Figure A3). Due to doubly-periodic supercell, we see multiple narrow bands bending 
down and crossing the band gap in Figure 1f. This is due to diffraction orders above the light line (white dotted line) [41] and does not affect the investigation of edge states, which follows.

\section{Circularly-Polarized Point Sources}

We begin by extending the investigation of our previous work [15] by exciting the system with point sources with circularly-polarized magnetic fields, $H=H_{x} \pm i H_{y}$. The directionality of modes is well understood for sources in the plane of the metasurface, $h=0$ : The inhomogeneous spin angular momentum of the edge eigenmode determines the direction of propagation, rather than the polarization of the source. This means it is possible to selectively couple to either of the counter-propagating edge states by moving the source in the plane of the metasurface; a result which holds for any bosonic system with this lattice, including photonic crystals [16] (we show this explicitly in Appendix D). In an experimental setup, it would be difficult to place sources in the plane of the metasurface. For example, a setup may have quantum dots or emitters, or a 2D material, placed on top of a spacing layer above the NPs in the metasurface. Therefore, it is important to characterise the coupling and directionality into the edge modes of sources that are outside of the metasurface place.

Experimentally, it is challenging to realise circularly-polarized magnetic dipoles at optical frequencies and in nanoscale setups, which would be required to excite directional modes in our metasurface by near-field sources. Previous experiments showed the coupling of Zeeman-induced circularly-polarized excited states of quantum dots to the directional edge modes in a photonic crystal [19]. However, the directionality in our metasurface is related to the circular polarisation of the in-plane magnetic field, meaning a magnetic rather than electric point source is required. At low frequencies, these sources have been realized by means of coaxial cables [20,42]. On the other hand, magnetic transitions in the optical regime can be realized with rare earth ions [43], although these are usually linearly-polarized transitions. A recent proposal makes use of two anti-parallel atomic dipoles to generate a magnetic dipole at optical frequencies [44,45], which could be extended to circular polarization, and possibly realized with quantum dots. Alternatively, the magnetic resonance of split-ring resonators could be engineered to provide the required source. In the following section, we assume a simple model and use a dielectric NP as source, given that they support a magnetic dipole mode [46], which could be excited with a circularly polarised wave. Provided the system can be modelled in the dipole approximation with subwavelength resonators and point dipole sources, the following results are valid regardless of the physical nature of the source or frequency regime. Therefore, our main conclusions also apply to metasurfaces made of microwave $[20,42]$ or Mie resonators [47].

The scattering setup is shown in Figure 2a. The source position is moved to various heights $h$ in the $z$-direction and the $x y$-position is fixed at the centre of the expanded unit cell, as shown in Figure 2a. The power through the left $\left(P_{L}\right)$ and right $\left(P_{R}\right)$ channels is calculated, as well as the power radiated by the source. The material losses along the interface are set to zero to test the directionality behaviour and they are gradually increased at the edge of the sample to prevent backscattering. Increasing material losses will not affect the directionality behaviour, but will affect how far the mode propagates. We show this explicitly in Appendix E, Figure A5.

We define a $\beta$-factor to characterise the amount of power coupling into edge modes compared to the total power radiated by the source,

$$
\beta_{i}=\frac{P_{i}}{P_{0} P_{F}},
$$

where $i=(L, R)$ corresponds to power through the left or right channel, respectively. $P_{0}$ is the power radiated by the source and $P_{F}$ is the Purcell factor. The total power coupling into the edge is then $\beta_{\text {edge }}=\beta_{\mathrm{L}}+\beta_{\mathrm{R}}$. If $\beta_{\text {edge }}=0$, none of the energy radiated from the source couples into the edge mode, whereas if $\beta_{\text {edge }}=1$ all of the energy couples into the edge. $\beta$ accounts for the enhancement of the 
emission of the source due to the environment through the Purcell factor [43]. For a magnetic dipole, the emitted power in free space,

$$
P_{0}=\mu_{0} \frac{\omega^{4}}{3 c^{3}}|\mathbf{m}|^{2}
$$

with vacuum permeability $\mu_{0}$ and magnetic dipole moment $\mathbf{m}$.

(a)

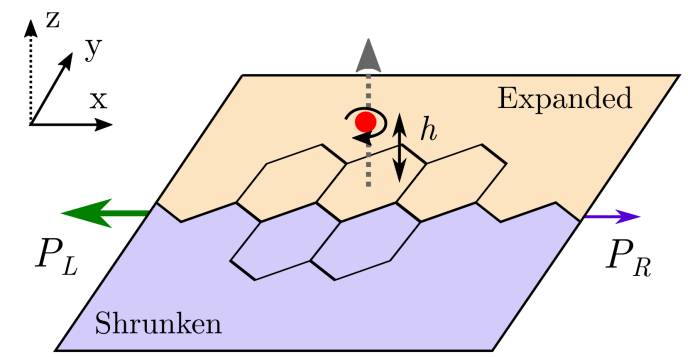

(b)

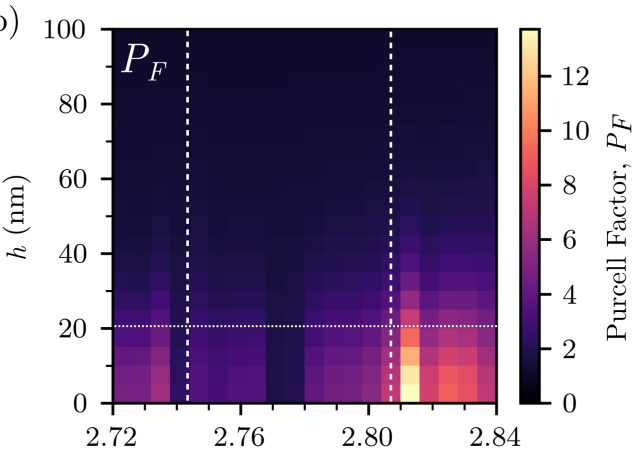

$\omega(\mathrm{eV})$

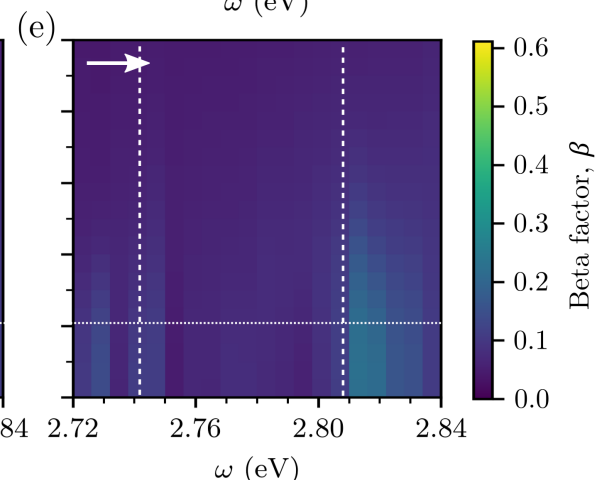

Figure 2. Single sources at varying heights $h$ and frequencies $\omega$ : (a) Schematic showing the layout of the interface. The system is 40 unit cells along $x$ and 12 unit cells along $y$, with 6 contracted and 6 expanded unit cells. A left circularly-polarized source (red) is placed at the centre of an expanded unit cell and moved upwards. The power through the left $P_{L}$ and right $P_{R}$ channels is used to characterise the directionality. (b) Purcell factor $P_{F}$ of the source. The height of the NPs is shown as a horizontal dotted line and the edges of the band gap are shown as vertical lines. Beta factors: (c) Power coupling into the edge $\beta_{\text {edge }}$ (double arrow). Power coupling into the (d) left $\beta_{L}$, and (e) right $\beta_{R}$ channels (left and right arrows).

The source is placed initially at $h=0 \mathrm{~nm}$ and then moved upwards to $h=100 \mathrm{~nm}$. We use a left circularly-polarized dipole throughout $\left(H=H_{x}-i H_{y}\right)$; which, in combination with the $x y$-position at the centre of the expanded unit cell, means we expect it to couple to the left propagating edge mode. Additionally, we scan over the frequency range of the band gap to investigate whether exciting in the upper or lower band has an effect on the directionality. We measure the normalised power through $y z$-planes perpendicular to both the metasurface and the interface. First, in Figure $2 \mathrm{~b}$ we plot the Purcell factor. (A horizontal white dashed line serves as a guide to the height of the NPs in the metasurface and the band gap is highlighted with vertical dashed lines). We see that the Purcell factor is greatest near the metasurface, since the array of NPs enhances the emission of the source by increasing the available local density of states (LDOS). For $h>50 \mathrm{~nm}$, there is almost no coupling to the metasurface and $P_{F} \approx 1$. Scanning across frequencies at $h$ close to zero, we can clearly see the minigap near $2.77 \mathrm{eV}$, where no NPs are excited, which causes $P_{F}$ to decrease. Lastly, $P_{F}$ is largest at $\omega \approx 2.81 \mathrm{eV}$ where the source begins to excite bulk modes. 
Next, in Figure 2c we plot $\beta_{\text {edge }}$ where it is clear that it is greatest for the edge state frequencies and smallest in the minigap and for bulk frequencies; in agreement with the spectral function of the edge states in Figure 1f. A maximum of approximately $60 \%$ of the power emitted by the source couples into the metasurface at $h=0$, and the rest radiates into free space as the source emits in all directions and the bulk of the metasurface is gapped. As the source is moved upwards, $\beta_{\text {edge }}$ decreases and follows the same relationship as $P_{F}$, such that, for $h>50 \mathrm{~nm}$, very little energy couples into the edge state. This confirms that the increase in $P_{F}$ is due to the source coupling to the edge. Finally, in Figure $2 \mathrm{~d}$,e we plot the beta factors for the left $\beta_{L}$ and right $\beta_{R}$ edge channels. As explained previously, since we use a left circularly-polarized source, which is initially placed at the centre of the unit cell, we expect and observe power flow predominantly through the left channel [15]-i.e., $\beta_{\mathrm{L}} \gg \beta_{\mathrm{R}}$. This pattern of directionality holds as the source is moved upwards.

Following the understanding of directionality for sources at different heights, we now investigate the position dependence in a plane parallel to the metasurface. The sources are placed in a range of positions in the $x y$-plane at a fixed height, $h=30 \mathrm{~nm}$, close to the interface, as shown in Figure 3a. We choose this height to ensure a $10 \mathrm{~nm}$ gap between the top of the NPs and the excitation layer, and to ensure enough power couples into the edge. In an experimental setup, this gap will be dependent on the type of source used. We will excite the system at $\omega=2.795 \mathrm{eV}$ and at $\omega=2.76 \mathrm{eV}$, and will refer to these from now on as the "upper band" and "lower band".

(a)

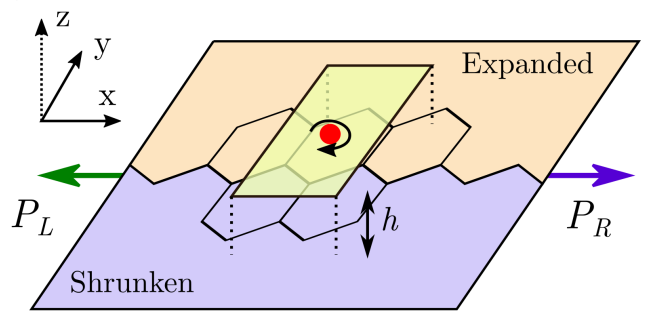

(d)
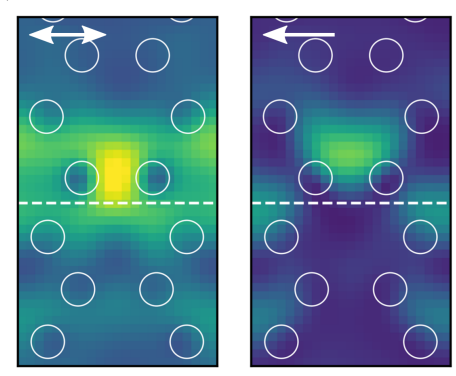

(b)

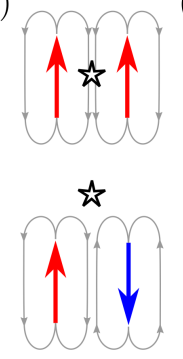

(c)
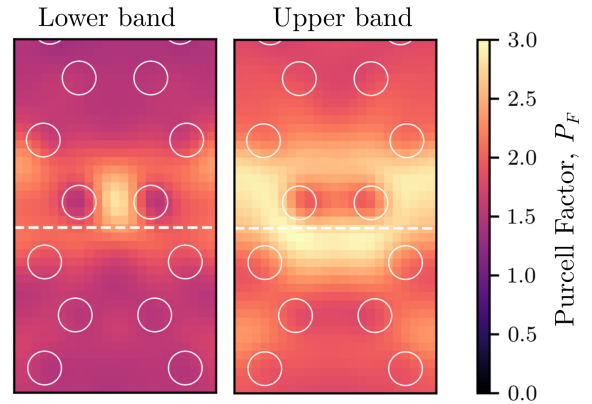

(e)
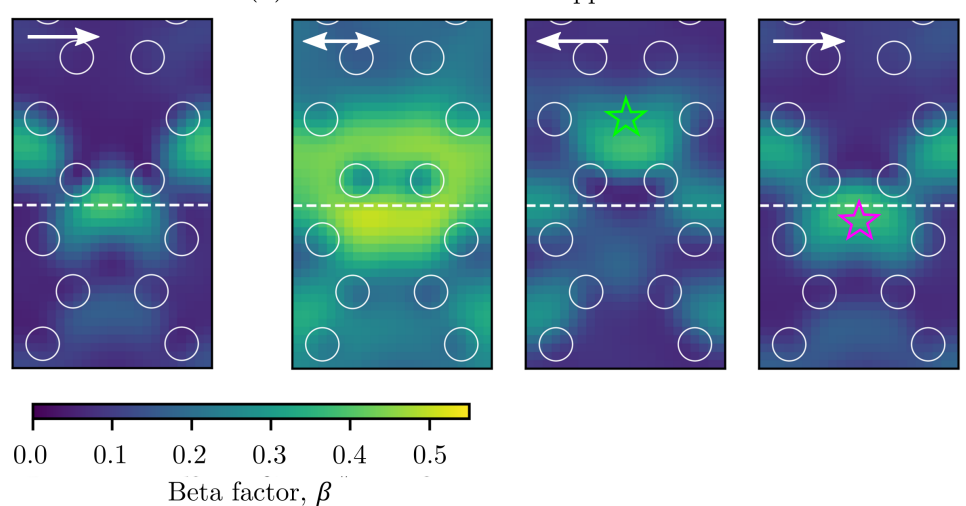

2
0
0
0
0
0
0
0
0
0
0
0 
In Figure 3c we plot the Purcell factor for the lower (left) and upper (right) bands: The pattern of each band is very different. From the ribbon eigenmodes in Figure $1 \mathrm{f}$ (and Appendix C, Figure A3), we showed that the upper and lower bands have a bonding and anti-bonding character, respectively. In Figure $3 b$ we sketch the out-of-plane dipole moments for the two NPs closest to the edge. This shows how the electric field maxima and minima will be in different positions. For the anti-bonding mode, there is a maximum (star) at some position above the NPs and for the bonding mode, the maximum is between the NPs. This is reflected in the peaks of the Purcell factor. Furthermore, for both bands, the Purcell factor is the greatest at the interface which, again, is due to the LDOS being the greatest here. Away from the interface, it approaches unity, but there is still some emission enhancement due to the proximity to the metasurface. However, this means that sources placed far from the interface will not excite edge modes.

Finally, in Figure 3d,e we plot the beta factors for the chosen frequencies in the lower and upper bands. $\beta_{\text {edge }}$ follows the same pattern as the Purcell factor. However, it is interesting to note that, despite the upper and lower bands having different $\beta_{\text {edge }}$ patterns, the position dependent directionality can still be seen in $\beta_{L}$ (middle panels) and $\beta_{R}$ (right panels). Specifically, for both bands, the coupling to the left propagating channel for a left circularly-polarized source is maximum when the source is above the centre of an expanded unit cell (lime star). As soon as the source is moved outside the hexamer of NPs (magenta star), it couples to the mode travelling in the opposite direction. For larger $h$, this pattern will eventually be lost as the source couples less and less to the edge state.

\section{Far-Field Circularly-Polarized Excitations}

A number of experimental works have investigated the directionality of these edge states under far-field excitation in a range of frequency regimes [17,21,22]. In each of these experiments, purely unidirectional modes are observed. Herein, we provide a detailed theoretical investigation of the directionality of edge modes with far-field excitations. We show that the position-dependent directionality also holds for far-field beams and that careful beam placement explains the unidrectionality observed in experiments. We investigate this behaviour by mimicking a circularly-polarized far-field Gaussian beam excitation with non-zero orbital angular momentum (OAM) incident on the metasurface. The NPs are excited with an incident electric field with a Gaussian intensity profile, $\mathbf{E}_{\mathrm{inc}}=\left(0,0, E_{z}\right)$ with,

$$
E_{z} \propto e^{-r^{2} /\left(2 w^{2}\right)} e^{i \phi}
$$

where $r$ is centre of the beam, $w$ is the full width at half maximum (FWHM), and $\phi$ is the angle in radians from the centre. The phase vortex in $E_{z}$ corresponds to a left or right circular polarization.

In Figure $4 \mathrm{a}$, we plot the phase of the $z$-component of the electric field of the edge eigenmodes: for the lower band, at $k_{x}>0$, and for the upper band, at $k_{x}<0$. A phase vortex structure is present across the interface, with clearly distinct regions at the centres of unit cells and at the edge of unit cells. Mode matching and maximum directionality occurs when the phase vortex of the beam rotates in the the same direction as the phase vortex of the edge eigenmode [48]. These positions are highlighted as clockwise arrows. Mode mismatch occurs when the beam and eigenmode have vortices rotating in opposite directions, and these positions are shown as anti-clockwise arrows. When there is a complete mode mismatch, the beam will excite a mode travelling in the opposite direction. The phase vortex behaviour of directional edge states has previously been studied in the context of valley modes [49-52], where valleys at $K$ and $K^{\prime}$ have vortices rotating in opposite directions. 
(a)

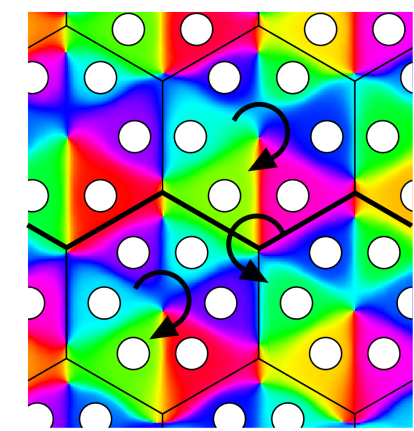

(c)

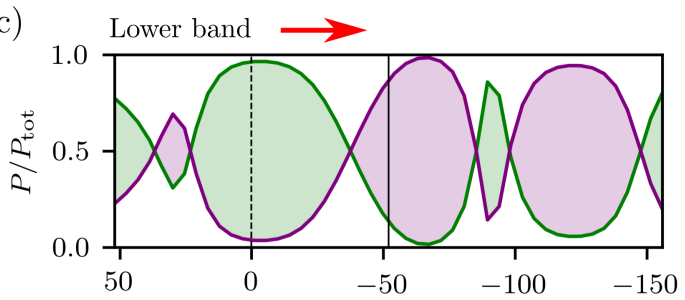

(e)

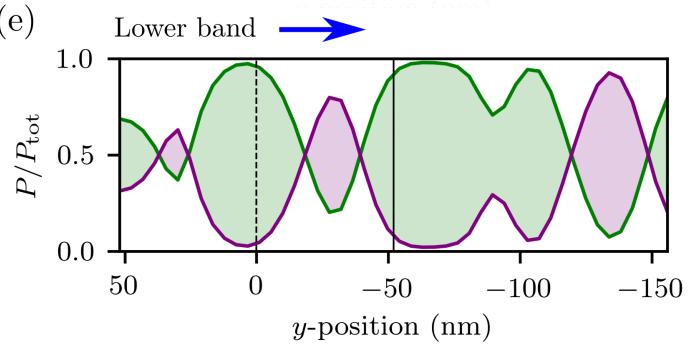

Upper band, $k_{x}<0$

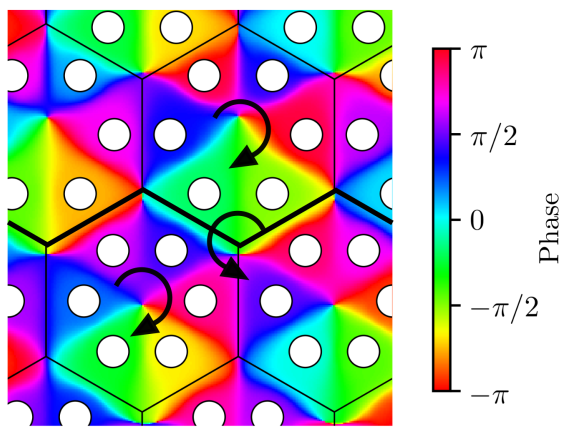

(b)

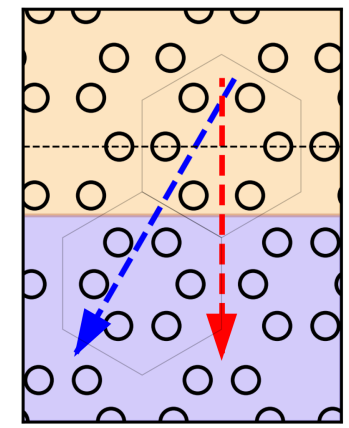

(d)
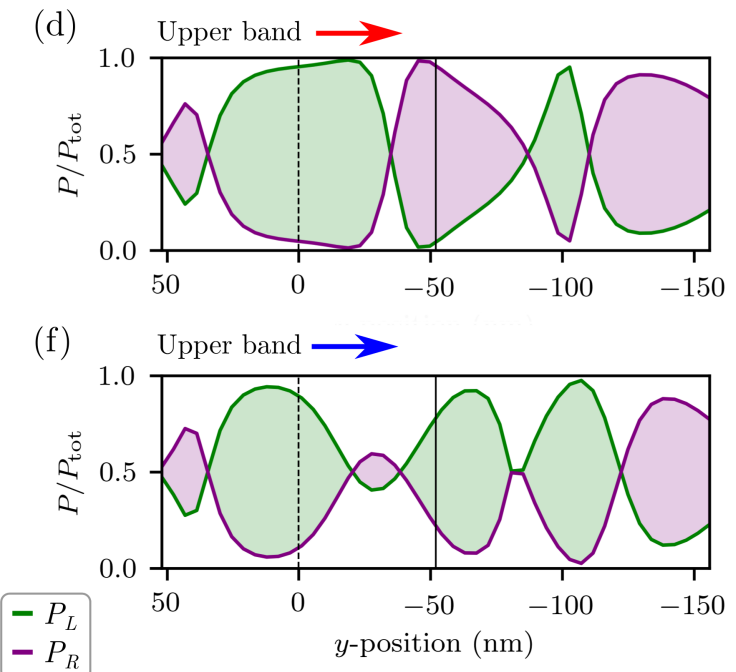

Figure 4. Far field excitations: (a) Phase of the $z$-component of the electric field for edge eigenmodes, in the (left) lower band for $k_{x}>0$ and (right) upper band for $k_{x}<0$. An incident beam with non-zero orbital angular momentum will couple to the phase vortices (black arrows) of the edge modes. (b) A diagram showing the paths of the beam across the interface between expanded (orange) and shrunken (blue) regions. The $y$-value of the centre of the expanded unit cell is shown as a dotted black line. (c,d) Beams along the red path. Fraction of power to the left $P_{L} / P_{\text {tot }}$ (green) and right $P_{R} / P_{\text {tot }}$ (purple) channels upper band $\omega=2.795 \mathrm{eV}$ and the lower band $\omega=2.76 \mathrm{eV}$. Regions are coloured to indicate directionality to the left or right. $y$-values for the centre of the unit cell (vertical dotted line) and interface (vertical solid line) are highlighted. (e,f) Beam along the blue path. Fraction of power to the left and right for lower and upper bands. This path is chosen to maximise directionality.

To confirm this behaviour we will probe the finite system with the beam in Equation (12). We let $w=50 \mathrm{~nm}$, meaning the beam covers approximately one unit cell and the centre of the beam is moved over the red and blue paths, shown in Figure $4 \mathrm{~b}$. As in the previous section, we excite the system at frequencies in the lower and upper bands at $\omega=2.76 \mathrm{eV}$ and $\omega=2.795 \mathrm{eV}$. In Figure 4c, $\mathrm{d}$ we plot the fraction of power through the left $\left(P_{L} / P_{\text {tot }}\right)$ and right $\left(P_{R} / P_{\text {tot }}\right)$ channels for the two bands (plots of the total power coupling to the edge modes in each case are shown in Appendix F). Importantly, when the centre of the beam is at the centre of the expanded unit cell $(y=0 \mathrm{~nm})$, the majority of power is through the left channel $P_{L} \gg P_{R}$, as was the case with point sources. Similarly, when the beam moves across the interface $(y \approx-50 \mathrm{~nm})$ the majority of power switches to the right channel $P_{L} \ll P_{R}$. Although we have only demonstrated this for two specific frequencies here, the position dependent directionality holds for frequencies across the band gap (as we show in Appendix F, Figure A6). The vortex map in Figure 4a shows that it is possible to choose a path which maximises directionality, by avoiding traversing over phase singularities. In Figure $4 \mathrm{~d}$ we plot the power for the blue path shown in Figure $4 \mathrm{a}$. This path is similar to scanning perpendicularly across a zig-zag interface, as in [21]. Compared to the 
red path, there is wider range of $y$-values that yield the expected directionality. Finally, we emphasise that it is the relationship between the FWHM and the lattice constant, rather than the FWHM value itself, which is important. For beams with a FWHM which is much larger than the lattice constant, directionality is mostly lost since the beam expands to cover and excite the majority of the interface.

In an experimental setup, edge modes in the metasurface could be excited with a combination of a 2D layer and far-field beam. For example, valley-selective modes can be excited using a transition metal dichalcogenide on top of plasmonic metasurface [53-55]. Along with the results from Section 4, the directionality observed for far-field excitations suggests that a similar method could be employed to excite directional modes in the breathing honeycomb lattice.

\section{Conclusions}

In this article, we have provided a comprehensive study of the excitation of edge modes in a plasmonic metasurface with a breathing honeycomb lattice arrangement. The 2D lattice of metallic NPs hosts subwavelength pseudospin edge modes which arise due to the topology of the bulk. The plasmonic metasurface is a versatile system for testing the directionality of these modes through the coupled dipole method.

Motivated by the excitation of chiral and valley selective modes in plasmonic metasurfaces with 2D layers, we probe the edge states of our system in the near- and far-field. With circularly-polarised magnetic dipole sources, we map the directionality of modes for sources out of the plane and show how, provided the source still couples to the interface, the pattern of directionality predicted by spin angular momentum is preserved. Additionally, we probe edge modes with far-field beams with non-zero orbital angular momentum. Here, the direction of propagation is predicted by the phase of the $E_{z}$ field of the edge eigenmodes.

Through our analysis, we show that the unidirectionality observed for experiments probing edge states in the breathing honeycomb lattice are reliant on careful placement of the source or beam excitation. Importantly, although we have particularized to the plasmonic metasurface, the directionality behaviour of the edge modes is applicable to any classical wave system possessing the same breathing honeycomb lattice.

Author Contributions: M.P. and P.A.H. conceptualized the project. M.P. wrote the code and performed the analysis. X.X. ran simulations for the photonic crystal. M.P. prepared the original draft. M.P., X.X., R.V.C., S.A.M., V.G. and P.A.H. contributed to reviewing the manuscript. All authors have read and agreed to the published version of the manuscript.

Funding: M.P., R.V.C and P.A.H. acknowledge funding from the Leverhulme Trust. V.G. acknowledges the Spanish Ministerio de Economia y Competitividad for financial support through the grant NANOTOPO (FIS2017-91413-EXP) and also the Ministerio de Ciencia, Innovación y Universidades through the grant MELODIA (PGC2018-095777-B-C21). P.A.H. acknowledges financial support from Fundação para a Ciência e a Tecnologia and Instituto de Telecomunicações under projects UID/EEA/50008/2020 and the CEEC Individual program with reference CEECIND/03866/2017. S.A.M. acknowledges the Lee-Lucas Chair in Physics.

Conflicts of Interest: The authors declare no conflict of interest.

\section{Abbreviations}

The following abbreviations are used in this manuscript:

2D Two Dimensional

FWHM Full Width at Half Maximum

LDOS Local Density of States

MWLWA Meier-Wokaun Long Wavelength Approximation

NP Nanoparticle

OAM Orbital Angular Momentum 


\section{Appendix A. Honeycomb Lattice: Optical Response of Bulk Modes}
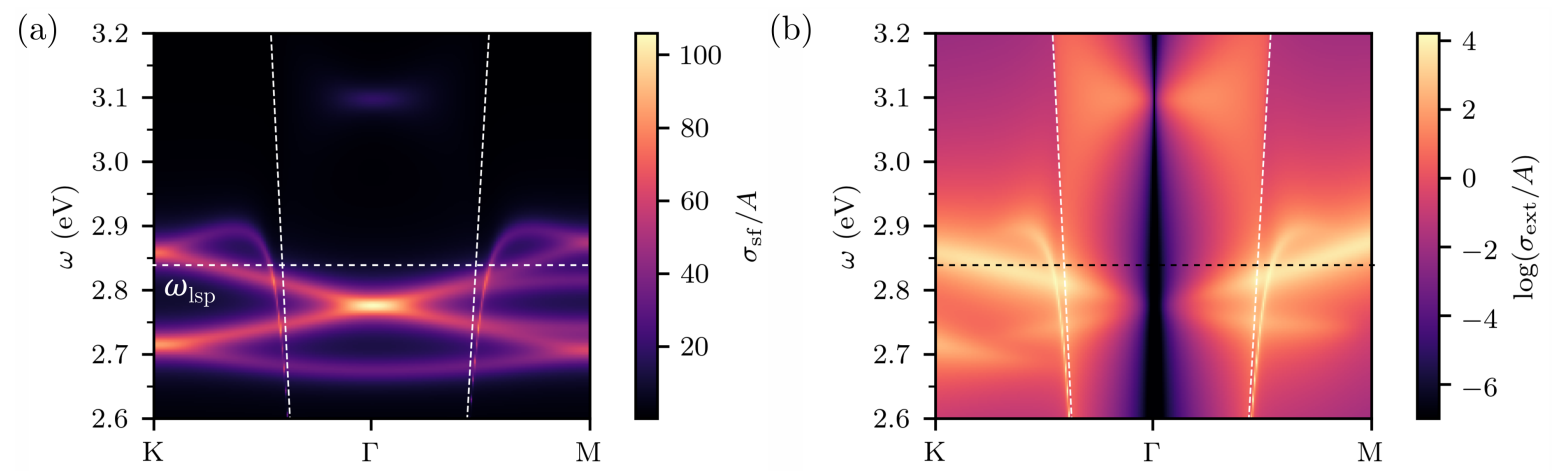

Figure A1. (a) Spectral function $\sigma_{\text {ext. }}$ There is a double Dirac cone degeneracy at $\Gamma$ due to the folded Brillouin zone, (b) Extinction cross section $\sigma_{\text {ext }}$.

\section{Appendix B. Edge States with Losses}

With the parameters in the main text, it would be difficult to observe edge states in a plasmonic metasurface ribbon with material losses $\gamma=38 \mathrm{meV}$. In Figure A2a, we plot the spectral function $\sigma_{\text {sf }}$ as in Figure $1 \mathrm{f}$ for $\gamma=38 \mathrm{meV}$. On the right, we plot $\sigma_{\text {sf }}$ for fixed parallel wavevector $k_{\|}$, shown as a vertical dotted line in the left panel. However, by tuning the shrunken and expanded perturbation factors, it is possible to increase and size of the band gap and have edge states which are distinguishable from the bulk. In Figure $\mathrm{A} 2 \mathrm{~b}$, we plot $\sigma_{\mathrm{sf}}$ for $R_{\mathrm{shr}}=0.85$ and $R_{\exp }=1.1$. With the larger band gap, the edge states are possible to distinguish from the bulk, as shown in the right panel.

(a) $R_{\mathrm{shr}}=0.9, R_{\exp }=1.065, \gamma=38 \mathrm{meV}$

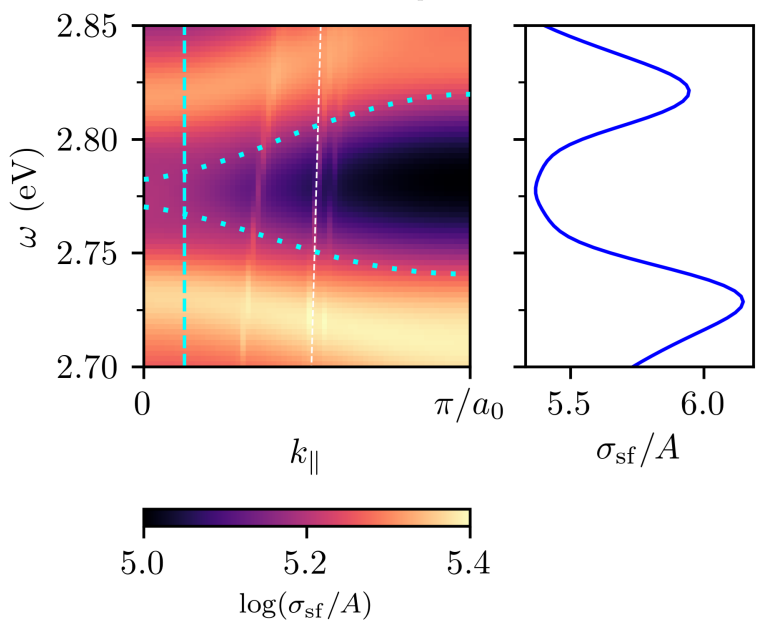

(b) $R_{\text {shr }}=0.85, R_{\text {exp }}=1.1, \gamma=38 \mathrm{meV}$

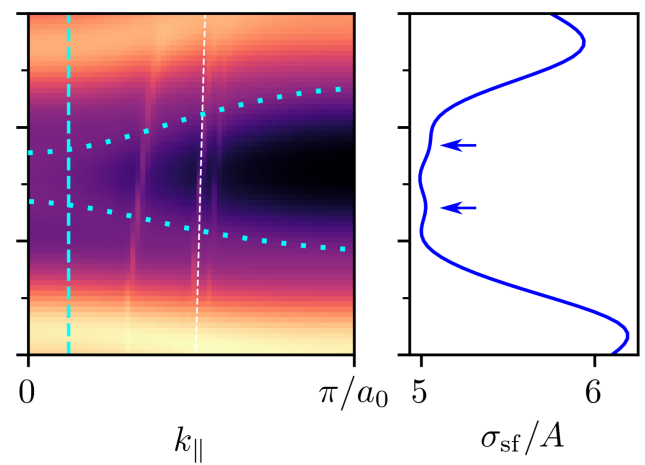

Figure A2. Spectral functions $\sigma_{\mathrm{sf}}$ for ribbons with material losses $\gamma=38 \mathrm{meV}$, with geometrical parameters (a) $R_{\mathrm{shr}}=0.9$ and $R_{\exp }=1.065$ and (b) $R_{\mathrm{shr}}=0.85$ and $R_{\exp }=1.1$.

\section{Appendix C. Edge State Eigenmodes}

The system of equations in Equation (6) for the ribbon interface is solved as an eigenvalue problem by letting $\mathbf{E}_{\mathrm{inc}}=0$. Additionally, the Green's function is linearised by letting $\omega=\omega_{\mathrm{lsp}}$, the localized surface plasmon frequency, in order to calculate dipole moments $\mathbf{p}$. The dipole moments for the armchair and zig-zag interfaces are shown in Figure A3. 
(a) Armchair

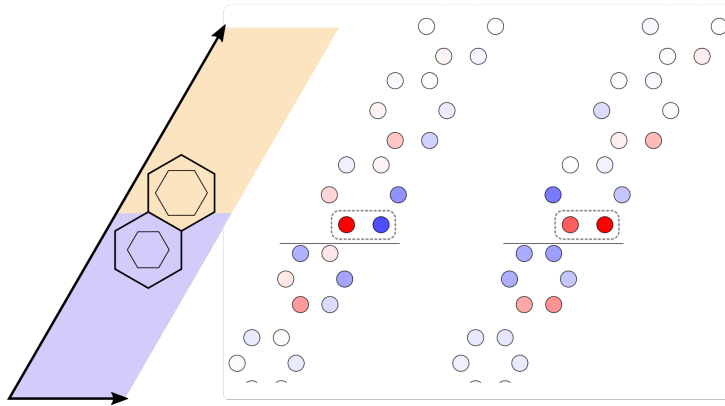

(b) Zig-zag

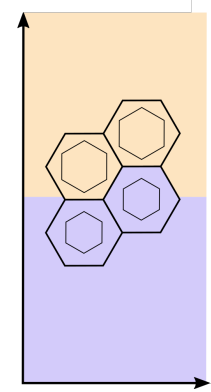

Lower Upper

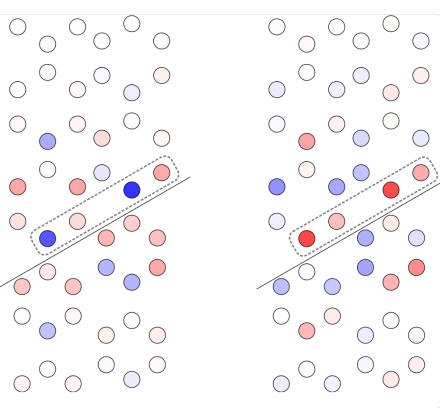

Figure A3. Hybridisation of NPs at interface: (a) Armchair interface, (b) Zig-zag interface.

\section{Appendix D. Directionality of Modes in a Photonic Crystal}

In the main text, we particularise to the subwavelength, plasmonic metasurface. We will show that the near-field position dependent directionality also holds in a photonic crystal, with constant permittivity, dielectric elements. We perform finite element simulations in COMSOL [56] on the same breathing honeycomb interface. The contracted region has $s=0.967$ and the expanded $s=1.041$, and the dielectric pillars are silicon with $\epsilon=11.7$ [12].

A left circularly-polarized magnetic dipole is used to excite modes at the three positions shown in Figure A4a. The electric field intensity $|\mathbf{E}|^{2}$ of the interface for the three source positions is shown Figure A4b. When the source is at the centre of the expanded unit cell (magenta star), it couples to a left propagating mode, whereas when the source is directly at the interface (green and cyan stars), it couples predominantly to a right propagating mode.

(a)

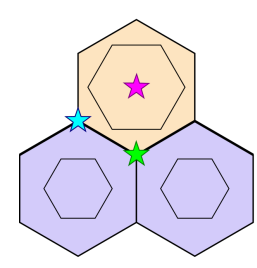

(b)

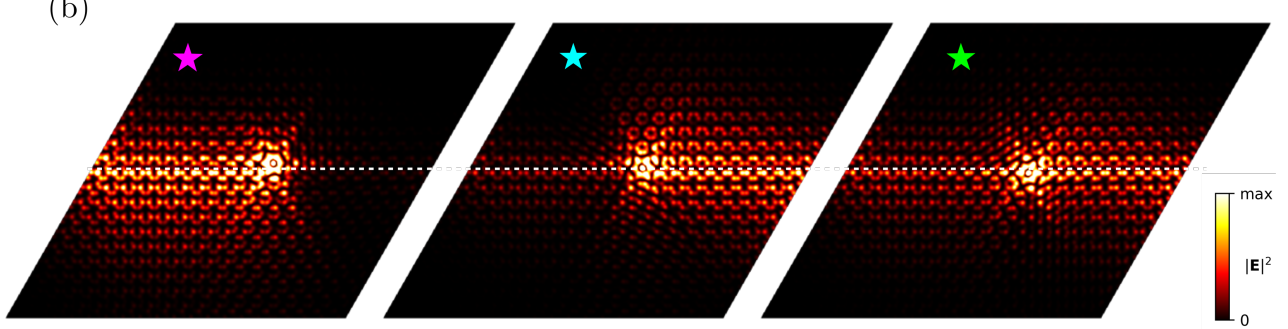

Figure A4. Breathing honeycomb photonic crystal interface. (a) Left circularly-polarised sources are placed at three positions near the interface. (b) Electric field intensity $|\mathbf{E}|^{2}$ for the different source positions, demonstrating source position dependent directionality.

\section{Appendix E. Propagation along the Interface with Losses}

Position-dependent directionality is not affected by near-field source position. To demonstrate this qualitatively, we excite the same interface as in the main text but with increasing material losses $\gamma$. For a left circularly polarised magnetic dipole source, in Figure A5a,b, we plot dipole moments at the lossless interface (for comparison), Figure A5c,d for $\gamma=10 \mathrm{meV}$ and Figure A5e,f for $\gamma=38 \mathrm{meV}$ (material losses). We see that, whilst increasing losses affects the propagation length of the modes, the directionality behaviour still holds. That is, for sources at the centre of the expanded unit cell Figure $\mathrm{A} 5 \mathrm{a}, \mathrm{c}, \mathrm{e}$ the propagation direction matches polarisation, and when the source is at the interface Figure $A 5 b, d, f$ the propagation direction switches. 
(a) $0 \mathrm{meV}, \mathrm{LCP}$, centre

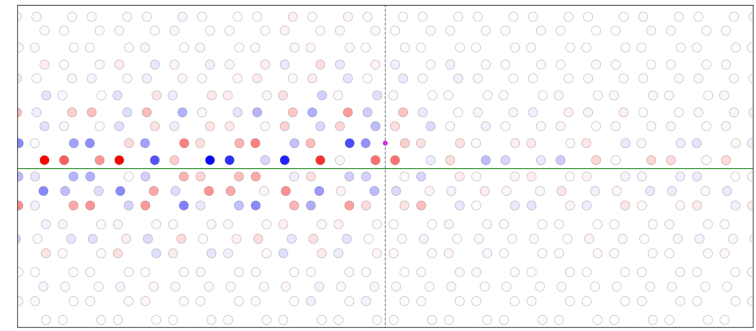

(c) $10 \mathrm{meV}$, LCP, centre

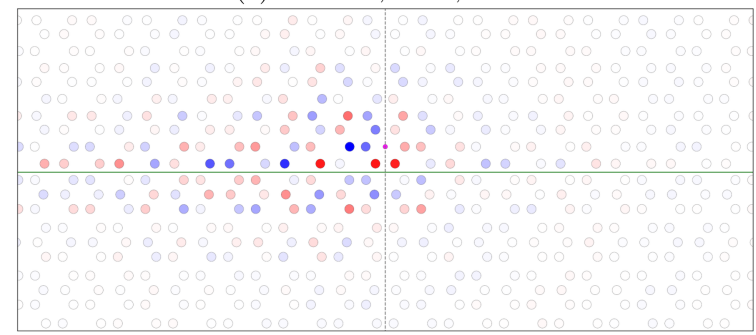

(e) $38 \mathrm{meV}$, LCP, centre

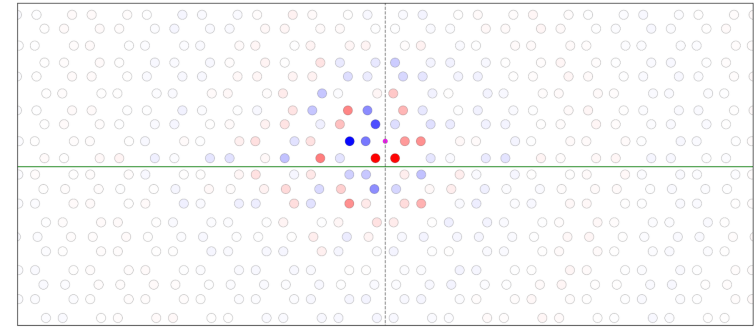

(b) $0 \mathrm{meV}$, LCP, edge

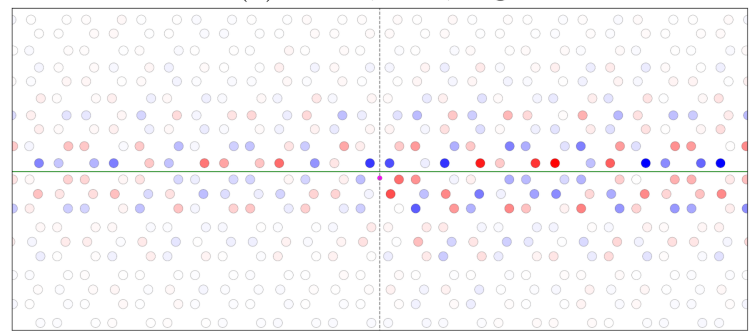

(d) $10 \mathrm{meV}$, LCP, edge

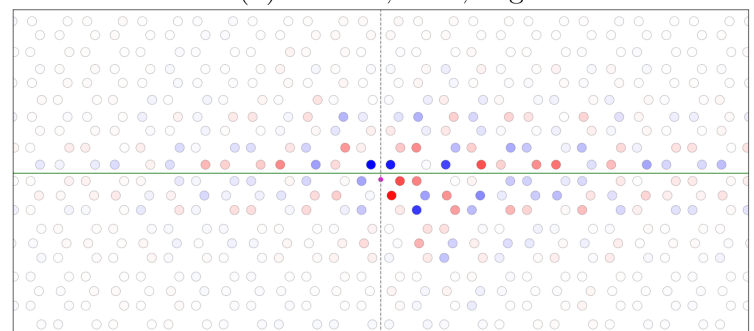

(f) $38 \mathrm{meV}$, LCP, edge

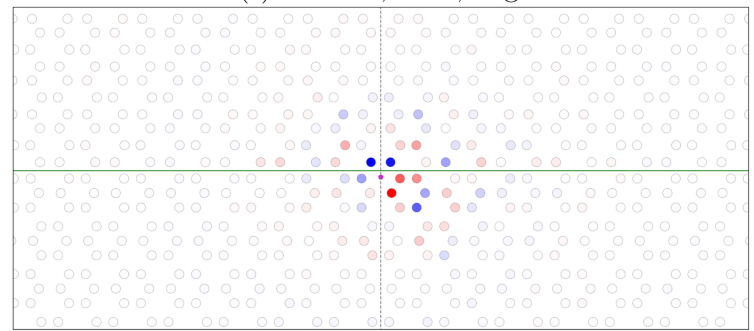

Figure A5. Propagation along lossy interfaces: Increasing material losses does not affect the directionality behaviour along the interface.

\section{Appendix F. Far Field Excitations: Power through Left and Right Channels}

Power through the left and right channels is calculated in the same way as in Section 5; however, here we plot the total power through the edge (normalised to the maximum) rather than the fraction left/right. As the beam moves downwards, we see how peaks in $P_{\text {tot }}$ follow a qualitatively similar pattern to the beta factors calculated in Figure $3 b$,d, which reflect the incident beam coupling to the edge modes. A maximum in $P_{\text {tot }}$ for the lower band occurs as the centre of the beam passes through the centre of two particles immediately at the interface, whereas for the upper band, $P_{\text {tot }}$ peaks either side of this. 
(a)

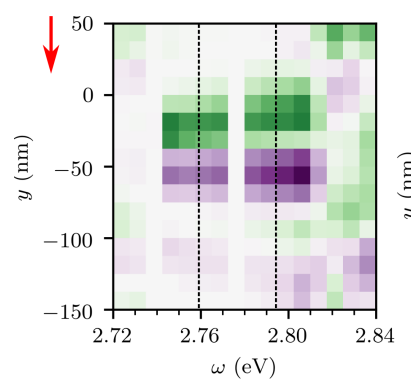

(b) Lower band

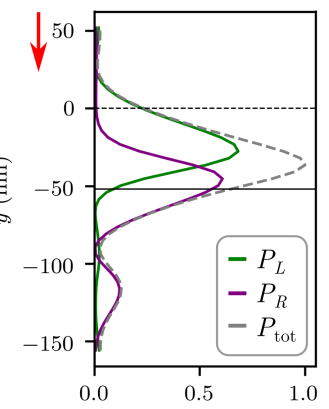

Upper band

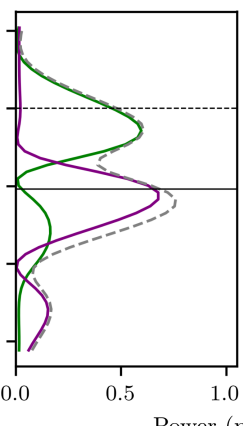

(c) Lower band

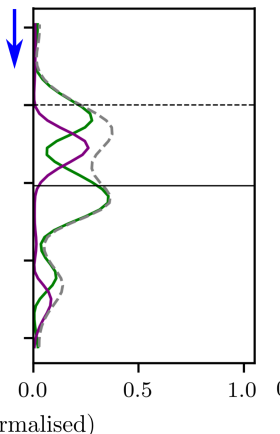

Upper band

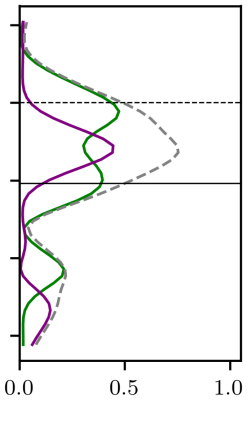

Figure A6. Far field excitations: (a) $P_{\Delta}=P_{L}-P_{R}$ for the red path in Figure $4 \mathrm{~b}$ and frequencies across the band gap. (b) Directionality along the red path: Power through the edge $P_{\text {tot }}$, left $P_{L}$ and right channels $P_{R}$ for lower and upper bands. (The lower and upper band line plots are equivalent to the vertical lines in (a).) (c) Directionality along the blue path.

\section{References}

1. Rider, M.S.; Palmer, S.J.; Pocock, S.R.; Xiao, X.; Arroyo Huidobro, P.; Giannini, V. A perspective on topological nanophotonics: Current status and future challenges. J. Appl. Phys. 2019, 125, 120901. [CrossRef]

2. Haldane, F.D.M.; Raghu, S. Possible Realization of Directional Optical Waveguides in Photonic Crystals with Broken Time-Reversal Symmetry. Phys. Rev. Lett. 2008, 100, 013904. [CrossRef] [PubMed]

3. Raghu, S.; Haldane, F.D.M. Analogs of quantum-Hall-effect edge states in photonic crystals. Phys. Rev. A 2008, 78, 033834. [CrossRef]

4. Wang, Z.; Chong, Y.; Joannopoulos, J.D.; Soljačić, M. Observation of unidirectional backscattering-immune topological electromagnetic states. Nature 2009, 461, 772-775. [CrossRef] [PubMed]

5. Jin, D.; Christensen, T.; Soljačić, M.; Fang, N.X.; Lu, L.; Zhang, X. Infrared Topological Plasmons in Graphene. Phys. Rev. Lett. 2017, 118, 245301. [CrossRef] [PubMed]

6. Pan, D.; Yu, R.; Xu, H.; García de Abajo, F.J. Topologically protected Dirac plasmons in a graphene superlattice. Nat. Commun. 2017, 8, 1243. [CrossRef] [PubMed]

7. Makwana, M.P.; Craster, R.V. Geometrically navigating topological plate modes around gentle and sharp bends. Phys. Rev. B 2018, 98, 184105. [CrossRef]

8. Wong, S.; Saba, M.; Hess, O.; Oh, S.S. Gapless unidirectional photonic transport using all-dielectric kagome lattices. Phys. Rev. Res. 2020, 2, 012011. [CrossRef]

9. Proctor, M.; Huidobro, P.A.; Maier, S.A.; Craster, R.V.; Makwana, M.P. Manipulating topological valley modes in plasmonic metasurfaces. Nanophotonics 2020, 9, 657-665. [CrossRef]

10. Saba, M.; Wong, S.; Elman, M.; Oh, S.S.; Hess, O. Nature of topological protection in photonic spin and valley Hall insulators. Phys. Rev. B 2020, 101, 054307. [CrossRef]

11. Orazbayev, B.; Fleury, R. Quantitative robustness analysis of topological edge modes in C6 and valley-Hall metamaterial waveguides. Nanophotonics 2019, 8, 1433-1441. [CrossRef]

12. Wu, L.H.; Hu, X. Scheme for Achieving a Topological Photonic Crystal by Using Dielectric Material. Phys. Rev. Lett. 2015, 114, 223901. [CrossRef] [PubMed]

13. De Paz, M.B.; Vergniory, M.G.; Bercioux, D.; García-Etxarri, A.; Bradlyn, B. Engineering fragile topology in photonic crystals: Topological quantum chemistry of light. Phys. Rev. Res. 2019, 1, 032005. [CrossRef]

14. Proctor, M.; Huidobro, P.A.; Bradlyn, B.; de Paz, M.B.; Vergniory, M.G.; Bercioux, D.; Garcia-Etxarri, A. On the robustness of topological corner modes in photonic crystals. arXiv 2020, arXiv:2007.10624.

15. Proctor, M.; Craster, R.V.; Maier, S.A.; Giannini, V.; Huidobro, P.A. Exciting Pseudospin-Dependent Edge States in Plasmonic Metasurfaces. ACS Photonics 2019, 6, 2985-2995. [CrossRef]

16. Oh, S.S.; Lang, B.; Beggs, D.M.; Huffaker, D.L.; Saba, M.; Hess, O. Chiral Light-matter Interaction in Dielectric Photonic Topological Insulators. In Proceedings of the CLEO Pacific Rim Conference 2018; Optical Society of America: Washington, DC, USA 2018; p. Th4H.5. [CrossRef]

17. Smirnova, D.; Kruk, S.; Leykam, D.; Melik-Gaykazyan, E.; Choi, D.Y.; Kivshar, Y. Third-Harmonic Generation in Photonic Topological Metasurfaces. Phys. Rev. Lett. 2019, 123, 103901. [CrossRef] 
18. Barik, S.; Miyake, H.; DeGottardi, W.; Waks, E.; Hafezi, M. Two-dimensionally confined topological edge states in photonic crystals. New J. Phys. 2016, 18, 113013. [CrossRef]

19. Barik, S.; Karasahin, A.; Flower, C.; Cai, T.; Miyake, H.; DeGottardi, W.; Hafezi, M.; Waks, E. A topological quantum optics interface. Science 2018, 359, 666-668. [CrossRef]

20. Yves, S.; Fleury, R.; Berthelot, T.; Fink, M.; Lemoult, F.; Lerosey, G. Crystalline metamaterials for topological properties at subwavelength scales. Nat. Commun. 2017, 8, 16023. [CrossRef]

21. Parappurath, N.; Alpeggiani, F.; Kuipers, L.; Verhagen, E. Direct observation of topological edge states in silicon photonic crystals: Spin, dispersion, and chiral routing. Sci. Adv. 2020, 6. [CrossRef]

22. Liu, W.; Hwang, M.; Ji, Z.; Wang, Y.; Modi, G.; Agarwal, R. Z 2 Photonic Topological Insulators in the Visible Wavelength Range for Robust Nanoscale Photonics. Nano Lett. 2020, 20, 1329-1335. [CrossRef] [PubMed]

23. Maier, S.A. Plasmonics: Fundamentals and Applications; Springer Science \& Business Media: Berlin/Heidelberg, Germany, 2007.

24. Weber, W.H.; Ford, G.W. Propagation of optical excitations by dipolar interactions in metal nanoparticle chains. Phys. Rev. B 2004, 70, 125429. [CrossRef]

25. Moroz, A. Depolarization field of spheroidal particles. J. Opt. Soc. Am. B 2009, 26, 517-527. [CrossRef]

26. Meier, M.; Wokaun, A. Enhanced fields on large metal particles: Dynamic depolarization. Opt. Lett. 1983, 8, 581-583. [CrossRef] [PubMed]

27. Yang, H.U.; D’Archangel, J.; Sundheimer, M.L.; Tucker, E.; Boreman, G.D.; Raschke, M.B. Optical dielectric function of silver. Phys. Rev. B 2015, 91, 235137. [CrossRef]

28. García de Abajo, F.J. Colloquium: Light scattering by particle and hole arrays. Rev. Mod. Phys. 2007, 79, 1267-1290. [CrossRef]

29. Wang, L.; Zhang, R.Y.; Xiao, M.; Han, D.; Chan, C.T.; Wen, W. The existence of topological edge states in honeycomb plasmonic lattices. New J. Phys. 2016, 18, 103029. [CrossRef]

30. Linton, C.M. Lattice Sums for the Helmholtz Equation. SIAM Rev. 2010, 52, 630-674. [CrossRef]

31. Kolkowski, R.; Koenderink, A.F. Lattice Resonances in Optical Metasurfaces With Gain and Loss. Proc. IEEE 2020, 108, 795-818. [CrossRef]

32. Koenderink, A.F.; Polman, A. Complex response and polariton-like dispersion splitting in periodic metal nanoparticle chains. Phys. Rev. B 2006, 74, 033402. [CrossRef]

33. Zhen, Y.R.; Fung, K.H.; Chan, C.T. Collective plasmonic modes in two-dimensional periodic arrays of metal nanoparticles. Phys. Rev. B 2008, 78, 035419. [CrossRef]

34. Pocock, S.R.; Xiao, X.; Huidobro, P.A.; Giannini, V. Topological Plasmonic Chain with Retardation and Radiative Effects. ACS Photonics 2018, 5, 2271-2279. [CrossRef]

35. Pocock, S.R.; Huidobro, P.A.; Giannini, V. Bulk-edge correspondence and long-range hopping in the topological plasmonic chain. Nanophotonics 2019, 8, 1337-1347. [CrossRef]

36. Merchiers, O.; Moreno, F.; González, F.; Saiz, J.M. Light scattering by an ensemble of interacting dipolar particles with both electric and magnetic polarizabilities. Phys. Rev. A 2007, 76, 043834. [CrossRef]

37. Gorlach, M.A.; Ni, X.; Smirnova, D.A.; Korobkin, D.; Zhirihin, D.; Slobozhanyuk, A.P.; Belov, P.A.; Alù, A.; Khanikaev, A.B. Far-field probing of leaky topological states in all-dielectric metasurfaces. Nat. Commun. 2018, 9, 909. [CrossRef]

38. Blanco de Paz, M.; Devescovi, C.; Giedke, G.; Saenz, J.J.; Vergniory, M.G.; Bradlyn, B.; Bercioux, D.; García-Etxarri, A. Tutorial: Computing Topological Invariants in 2D Photonic Crystals. Adv. Quantum Technol. 2020, 3, 1900117. [CrossRef]

39. Lodahl, P.; Mahmoodian, S.; Stobbe, S.; Rauschenbeutel, A.; Schneeweiss, P.; Volz, J.; Pichler, H.; Zoller, P. Chiral quantum optics. Nature 2017, 541, 473-480. [CrossRef]

40. Kariyado, T.; Hu, X. Topological States Characterized by Mirror Winding Numbers in Graphene with Bond Modulation. Sci. Rep. 2017, 7, 16515. [CrossRef]

41. Cherqui, C.; Bourgeois, M.R.; Wang, D.; Schatz, G.C. Plasmonic Surface Lattice Resonances: Theory and Computation. Accounts Chem. Res. 2019, 52, 2548-2558. [CrossRef]

42. Yves, S.; Berthelot, T.; Lerosey, G.; Lemoult, F. Locally polarized wave propagation through crystalline metamaterials. Phys. Rev. B 2020, 101, 035127. [CrossRef]

43. Baranov, D.G.; Savelev, R.S.; Li, S.V.; Krasnok, A.E.; Alù, A. Modifying magnetic dipole spontaneous emission with nanophotonic structures. Laser Photonics Rev. 2017, 11, 1600268. [CrossRef] 
44. Alaee, R.; Gurlek, B.; Albooyeh, M.; Martín-Cano, D.; Sandoghdar, V. Quantum Metamaterials with Magnetic Response at Optical Frequencies. Phys. Rev. Lett. 2020, 125, 063601. [CrossRef] [PubMed]

45. Ballantine, K.E.; Ruostekoski, J. Optical Magnetism and Huygens' Surfaces in Arrays of Atoms Induced by Cooperative Responses. arXiv 2020, arXiv:2002.12930.

46. García-Etxarri, A.; Gómez-Medina, R.; Froufe-Pérez, L.S.; López, C.; Chantada, L.; Scheffold, F.; Aizpurua, J.; Nieto-Vesperinas, M.; Sáenz, J.J. Strong magnetic response of submicron Silicon particles in the infrared. Opt. Express 2011, 19, 4815-4826. [CrossRef] [PubMed]

47. Kim, M.; Rho, J. Quantum Hall phase and chiral edge states simulated by a coupled dipole method. Phys. Rev. B 2020, 101, 195105. [CrossRef]

48. Deng, W.M.; Chen, X.D.; Zhao, F.L.; Dong, J.W. Transverse angular momentum in topological photonic crystals. J. Opt. 2017, 20, 014006. [CrossRef]

49. Deng, W.M.; Chen, X.D.; Chen, W.J.; Zhao, F.L.; Dong, J.W. Vortex index identification and unidirectional propagation in Kagome photonic crystals. Nanophotonics 2019, 8, 833-840. [CrossRef]

50. Chen, X.D.; Zhao, F.L.; Chen, M.; Dong, J.W. Valley-contrasting physics in all-dielectric photonic crystals: Orbital angular momentum and topological propagation. Phys. Rev. B 2017, 96, 020202. [CrossRef]

51. Chen, X.D.; Shi, F.L.; Liu, H.; Lu, J.C.; Deng, W.M.; Dai, J.Y.; Cheng, Q.; Dong, J.W. Tunable Electromagnetic Flow Control in Valley Photonic Crystal Waveguides. Phys. Rev. Appl. 2018, 10, 044002. [CrossRef]

52. Ye, L.; Yang, Y.; Hang, Z.H.; Qiu, C.; Liu, Z. Observation of valley-selective microwave transport in photonic crystals. Appl. Phys. Lett. 2017, 111, 251107. [CrossRef]

53. Chervy, T.; Azzini, S.; Lorchat, E.; Wang, S.; Gorodetski, Y.; Hutchison, J.A.; Berciaud, S.; Ebbesen, T.W.; Genet, C. Room Temperature Chiral Coupling of Valley Excitons with Spin-Momentum Locked Surface Plasmons. ACS Photonics 2018, 5, 1281-1287. [CrossRef]

54. Hu, G.; Hong, X.; Wang, K.; Wu, J.; Xu, H.X.; Zhao, W.; Liu, W.; Zhang, S.; Garcia-Vidal, F.; Wang, B.; et al. Coherent steering of nonlinear chiral valley photons with a synthetic $\mathrm{Au}-\mathrm{WS}_{2}$ metasurface. Nat. Photonics 2019, 13, 467-472. [CrossRef]

55. Sun, L.; Wang, C.Y.; Krasnok, A.; Choi, J.; Shi, J.; Gomez-Diaz, J.S.; Zepeda, A.; Gwo, S.; Shih, C.K.; Alù, A.; et al. Separation of valley excitons in $\mathrm{MoS}_{2}$ monolayer using a subwavelength asymmetric groove array. Nat. Photonics 2019, 13, 180-184. [CrossRef]

56. COMSOL AB, Stockholm. RF Module-COMSOL Multiphysics, v. 5.4. Available online: https://uk.comsol. com/support/knowledgebase/1223 (accessed on 27 August 2020).

(C) 2020 by the authors. Licensee MDPI, Basel, Switzerland. This article is an open access article distributed under the terms and conditions of the Creative Commons Attribution (CC BY) license (http:/ / creativecommons.org/licenses/by/4.0/). 